\title{
Fabrication and Application of Phase only Holograms for High Power Laser Beam Shaping
}

\author{
Ilya Alexeev ${ }^{1,2}$, Eric Brehm ${ }^{1}$, Florian Nendel ${ }^{1}$, Johannes Strau $\beta^{1,2}$, Marcus Baum ${ }^{1,2}$, Michael Schmidt ${ }^{1,2}$ \\ ${ }^{1}$ Institute of Photonic Technologies, Friedrich-Alexander-University of Erlangen-Nuremberg, Konrad-Zuse-Str. 3, 91052 \\ Erlangen, Germany \\ E-mail: ilya.alexeev@lpt.uni-erlangen.de \\ ${ }^{2}$ Erlangen Graduate School in Advanced Optical Technologies (SAOT), Friedrich-Alexander-University of Erlangen- \\ Nuremberg, Paul-Gordan-Str. 6, 91052 Erlangen, Germany
}

\begin{abstract}
Fabrication of phase only holograms via spatially selective ablation of an ITO nanoparticulate layer coated on an optical quality transparent substrate appears to be very attractive due to the simplicity and flexibility of this approach. A pre-calculated binary pattern can be directly written on such a layer in order to produce a desired intensity distribution in the focal plane of a focusing lens. In this contribution we analyze the performance of the fabricated holograms and their suitability for high power beam shaping as well as propose a way to improve the diffraction efficiency of such structures by transitioning from a single to multi-layer holograms.
\end{abstract}

DOI: $10.2961 /$ jlmn.2016.02.0019

Keywords: phase only holograms, ultrafast ablation, ITO nanoparticles, beam shaping

\section{Introduction}

Nowadays computer generated holograms play an important role in many fields spanning from consumer applications [1] to highly scientific use [2]. Such holograms can be realized either via addressable dynamic devices such as spatial light modulators [3] or via fixed structures commonly called diffractive optical elements (DOEs). There are a number of ways that are currently used for DOE fabrication. Although the lithography based processes followed by an etching step [4] are probably the most common approach, mechanical fabrication methods based on turning [5], [6], injection molding and hot embossing [7], [8] have also been demonstrated. Fabrication processes based on direct laser ablation of bulk material [8] are appealing due to their flexibility but the achieved surface roughness is not sufficient to ensure required phase shifts at visible wavelengths. Recently, a structuring method based on spatially selective ultrafast laser ablation of an indium tin oxide (ITO) nanoparticulate layer has been proposed and investigated for Fraunhofer holograms [10]. An ITO nanoparticulate layer of thickness up to $1 \mu \mathrm{m}$ has been shown to be highly transparent in the visible and near infrared spectrum [11]. Such a layer can be spin-coated on a glass substrate with the resulting surface quality of the deposited layer to be only slightly inferior to the surface quality of the underlying optical substrate. The layer thickness is controllable via the density of the ITO suspension used for coating [12]. Knowing the refractive index of the ITO nanoparticle coating [11] the layer thickness $T$ is adjusted to achieve a phase retardation of $\pi$ between the waves traveling an identical physical distance in air and in the coating: $T=\frac{\lambda}{2\left(n_{\lambda}-1\right)}$, where $\lambda$ is the wavelength of interest and $n_{\lambda}$ is the corresponding refractive index. The laser ablation thresholds of the ITO coating and the glass substrate are vastly different $\left(\sim 0.63 \mathrm{~J} / \mathrm{cm}^{2}\right.$ and $4.2 \mathrm{~J} / \mathrm{cm}^{2}$ respectively [10]) allowing very clean spatially selective removal of the ITO layer without damaging the underlying transparent base. The latter permits laser ablation of pixelated structures without compromising the overall optical quality. In order to fabricate a binary $(0, \pi$ phase shift) diffractive optical element a precalculated pixelated structure has to be directly written (ablated) on an ITO nanoparticle coated substrate. The simplicity, high speed, and low cost of fabrication of these binary Fraunhofer holograms make them appealing to a number of applications such as shaping of high power $\mathrm{cw}$ lasers. In those applications use of dynamic shaping devices such as SLMs is highly restricted due to the high thermal load, while fabrication of various static DOEs using the lithographic methods can be too costly. If such ITO nanoparticle holograms are to be used for laser beam shaping their ability to withstand high laser intensities in the cw mode must be demonstrated along with increased diffraction efficiency. The demonstrated efficiency of $28 \%$ per $\pm 1^{\text {st }}$ diffraction order [10] appears to be significantly lower than the theoretical maxim of $40.5 \%$.

In this contribution we investigate ability of ITO nanoparticulate layers and holograms to withstand high cw laser intensities. We also describe a fabrication method used to improve the diffraction efficiency of the holograms.

\section{Hologram fabrication}

In the presented work the authors used ITO nanoparticles commercially available from Evonik Industries AG with the primary particle size being about $20 \mathrm{~nm}$. An ITO suspension had been prepared by dispersing the nanoparticles in ethanol at a $20 \mathrm{wt} \%$ loading. 2-[2-(2-Methoxyethoxy) ethoxy] acetic acid (also known as trioxydecanoic acid) here acting as a steric stabilizer had been added to the suspension to minimize particle agglomeration. $200 \mu \mathrm{l}$ of the base suspension was spin-coated on a glass substrate at 
a rate of $3000 \mathrm{rpm}$ for 20 seconds to establish the base thickness of the coating. Then the thickness of a new layer can be either reduced by dilution the base suspension with ethanol or increased via multiple coating runs. In general, it is preferable to keep the number of coating runs as few as possible since each subsequent layer increases the surface roughness $R_{a}$ by approximately $1 \mathrm{~nm}$ with the first coating having $R_{a}$ of $4 \mathrm{~nm}$ over a cut-off distance $\lambda_{c}$ of $0.08 \mathrm{~mm}$. Three types of glass substrates were used in the study: $25 \mathrm{~mm} \times 25 \mathrm{~mm} \times 1 \mathrm{~mm}$ soda lime microscope slides from Carl Roth GmbH, GE 124 fused quartz slides from TED PELLA, Inc., and a $1 \mathrm{~mm}$ thick $25.4 \mathrm{~mm}$ in diameter fused silica window broadband AR coated for $350-700 \mathrm{~nm}$ from Thorlabs, Inc. In order to achieve localized ablation of the ITO nanoparticle layers, we used a femtosecond laser (Spectra Physics Spitfire XP) with a wavelength of $800 \mathrm{~nm}$, a pulse length of $100 \mathrm{fs}$, and a repetition rate of $1 \mathrm{kHz}$. The laser pulse energy was empirically adjusted for a given focusing geometry to achieve clean ablation of the ITO coating without damaging the underlying glass substrate. Typically three laser pulses per ablated area were used to ensure complete removal of the nanoparticles within the spot. Two focusing geometries of the structuring ultrafast laser beam were applied during the experiment. In the first configuration a $60 \mathrm{~mm}$ focal length lens was used to achieve ablation spots of $13-35 \mu \mathrm{m}$ in diameter depending on the size of the structuring beam (adjusted by an aperture in front of the focusing lens). In the second configuration a mask with a square shaped cutout of $570 \mu \mathrm{m}$ (side) was introduced into the beam path and reimaged with a Nikon microscope objective (LWD 20x/0.40) on a sample to be structured. In the latter setup square shaped pixels with a side length of $14.5 \mu \mathrm{m}$ were ablated from the sample. The hologram binary patterns were calculated using a commercial software package VirtualLab with the overall size typically restricted to $300 \times 300$ pixels to reduce the processing time. During the fabrication phase the laser focus remained stationary with the sample was translated in the orthogonal $x-y$ plane with a 2D linear stage. Produced holograms and ablated structures were examined with a laser scanning microscope (LSM, Olympus, Lext OLS4000).

\section{Applicability for high power beam shaping}

Deposited ITO nanoparticulate layers have low absorption in the spectral region below $1 \mu \mathrm{m}$ but it starts to rapidly increase after that. The transmission window can be significantly broadened via sample annealing in air at temperatures around $450^{\circ}$ for approximately $30 \mathrm{~min}$ [13]. The annealing process leads to the oxidization of the material and thus reduction of the number of oxygen vacancies resulting in less free electrons. The plasma edge becomes red-shifted noticeably increasing the transmission in the spectral range $1.0-1.5 \mu \mathrm{m}$. Fig. 1 shows transmission of a bare fused silica substrate and an annealed ITO nanoparticle layer coated on a similar fused silica slide in a spectral range of $0.9-1.2 \mu \mathrm{m}$. In the presented case the ITO layer thickness was $1600 \mathrm{~nm}$ to achieve a phase shift of $\pi$ for a wavelength around $1 \mu \mathrm{m}$ (4 subsequent coating runs were performed). As it can be seen from the figure the absorption in the range $1.0-1.1 \mu \mathrm{m}$ rises only marginally, potentially making this type of holograms suitable for beam shaping of modern high power cw solid state and fiber lasers emitting $1.030-1.070 \mu \mathrm{m}$ wavelengths.

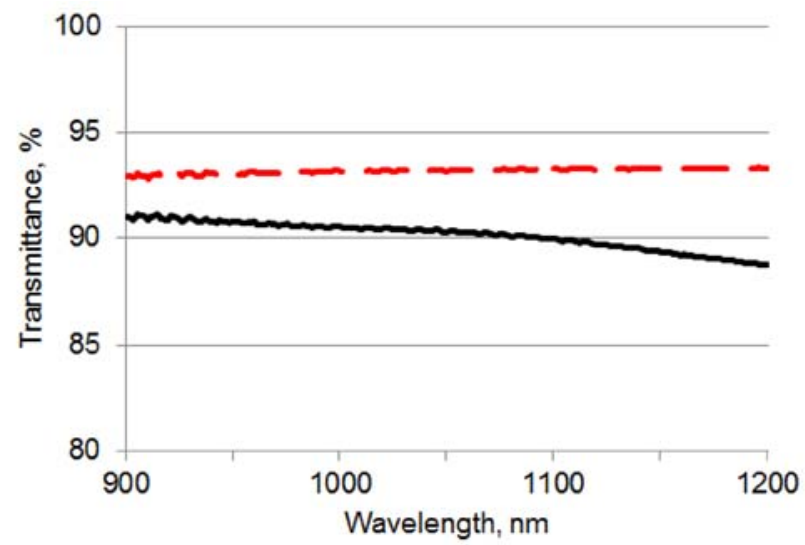

Fig.1 Transmittance of a bare fused silica glass substrate (red dashed line) and an annealed ITO coated $1600 \mathrm{~nm}$ thick (black solid line) fused silica sample.

To evaluate durability of ITO coatings a layer of comparable thickness was deposited on several fused silica substrates that were subsequently annealed and irradiated with a high power cw laser beam emitted by a $3.9 \mathrm{~kW}$ disk laser TruDisk 4002 (Trumpf GmbH). The laser wavelength is $1.030 \mu \mathrm{m}$ and the beam was defocused to a diameter of $15 \mathrm{~mm}$. Assuming a Gaussian intensity distribution in the transvers beam profile, the stated laser parameters correspond to peak $\mathrm{cw}$ intensity of $\sim 4 \mathrm{~kW} / \mathrm{cm}^{2}$. The durability testing was performed with a number of ON/OFF cycles to mimic a typical operation mode of high power lasers. An uncooled sample was able to withstand three 20 seconds ON 20 seconds OFF cycles at a laser power level of 1.5 $\mathrm{kW}$. The applied laser cycles were unable to shatter the test sample or crack the coating layer although small delamination was sometimes observed around big agglomerates (appear as black dots in Fig. 2). The areas without agglomerates did not show any layer delamination (Fig. 2) leading us to conclude that the quality of the ITO nanoparticle suspension will strongly influence the coating damage threshold.

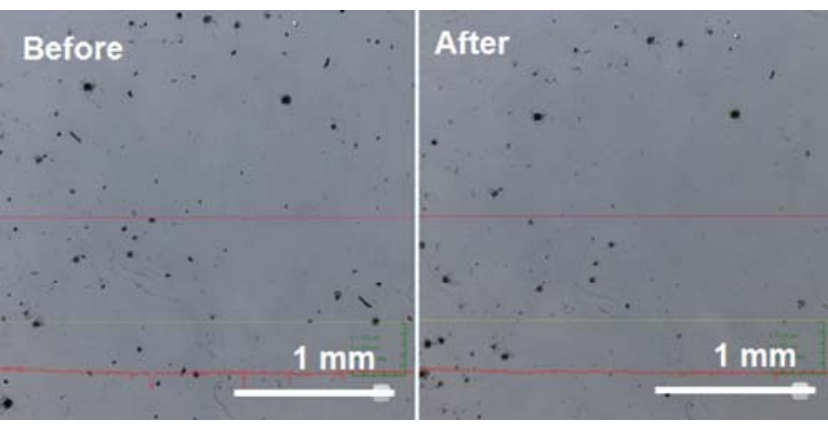

Fig. 2 Sample images of an ITO nanoparticulate layer before and after the durability test. The layer thickness is around $1600 \mathrm{~nm}$. No apparent damage to the layer is visible.

A similar uncooled sample also survived irradiation at the full laser power for 3 seconds although further testing demonstrated that longer exposure $(\sim 30 \mathrm{sec})$ eventually 
leads to test sample destruction. Cooling of the samples with compressed air allowed them not to be destroyed at least for 60 seconds.

\section{Beam shaping with binary phase only holograms}

Although the ability of ITO coatings to withstand high cw power levels is crucial for the envisioned applications it is equally important to evaluate the diffraction efficiency as well as beam shaping capabilities of such holograms. The reported diffraction efficiency of $28 \%$ [10] appears to be too low and must be increased for any shaping application besides demonstration of visual effects. The diffraction efficiency can be increased via modifying the fabrication process. Firstly, the hologram design software implicitly presumes that the pixels have a square shape since the optimization algorithm is based on a discrete 2D Fourier transform. As a result square shaped pixels must be also ablated in the ITO coating to match the simulated mask. In the previous work ablated pixels had a circular shape. Secondly, antireflective coated substrates can be used to reduce the parasitic Fresnel back reflection from the hologram. Although such AR coatings should not improve the relative diffraction efficiency of a phase only hologram they will increase the overall transmission. Moreover, combining the first and the minus first diffraction orders will not only allow the on-axis operation but will also double the diffraction efficiency. To remind the reader the maximum diffraction efficiency for a binary grating is $40.5 \%$ per $\pm 1^{\text {st }}$ order [14] potentially allowing to achieve $81 \%$ efficiency for a diffraction order combining hologram. The overlapping of the positive and negative orders will limit the set of possible beam shapes but since some sort of symmetry in the beam intensity distribution is almost always present such a limitation is acceptable. In order to quantify the proposed modifications a $250 \times 250$ pixel hologram was calculated for the design wavelength of $0.532 \mu \mathrm{m}$ (layer thickness $530 \mathrm{~nm}$ ). The hologram was calculated to reshape a $1.2 \mathrm{~mm} 1 / e^{2}$ radius Gaussian laser beam into a flat-top profile with a $300 \mu \mathrm{m} 1 / e^{2}$ radius in the focal plane of a $400 \mathrm{~mm}$ lens. Here, the flat-top distribution was simulated as a super Gaussian function with $m=10$ ( $m=1$ corresponds to a Gaussian beam).

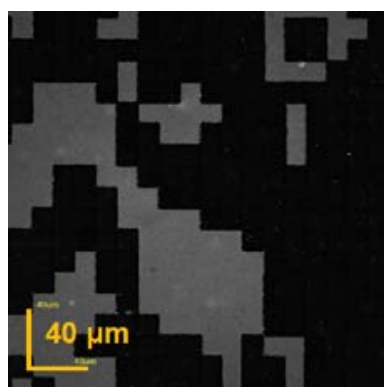

Fig. 3 A fragment of the ablated hologram. The pixel size is $14.5 \mu \mathrm{m}$ with a matching pixel pitch. The layer thickness is $530 \mathrm{~nm}$. The substrate is a fused silica 1 mm thick window AR coated for $350-700 \mathrm{~nm}$ on both sides.

Fig. 3 shows a fragment of an order combing hologram ablated on the double side AR coated window. The hologram clearly demonstrates high quality ablation of square pixels. The ablated area appears black due to the AR coating which was left absolutely undamaged.

The measured on-axis diffraction efficiency of the ablated hologram was $77 \%$ relative and $73 \%$ absolute. Here, the relative efficiency is defined as a ratio of the combined laser power in the $\pm 1^{\text {st }}$ orders to the total laser power transmitted through the hologram. The obtained value is very close to the theoretical maximum of $81 \%$ and represents significant improvement over the earlier result of $56 \%$ efficiency (both numbers are for the combined diffraction efficiency of the $\pm 1^{\text {st }}$ orders). The absolute efficiency is defined as a ratio of the combined laser power in the $\pm 1^{\text {st }}$ orders to the input laser power before the hologram. The difference of $4 \%$ indicates the Fresnel reflection was indeed reduced compared to the bare glass substrate but was not completely eliminated. A proper choice of the AR coating can increase the absolute efficiency of a hologram and reduce parasitic back reflection primarily from the structured surface.

Fig. 4a shows the simulated and measured beam profiles in the focal plane of the focusing lens, while Fig. $4 \mathrm{~b}$ shows the corresponding intensity outlines in the two orthogonal directions (measured). A clear match between the beam sizes and the overall intensity distribution can be easily seen in the figure. For binary holograms the zero diffraction order should be nonexistent and it is also not pronounced in the recorded image.

a)
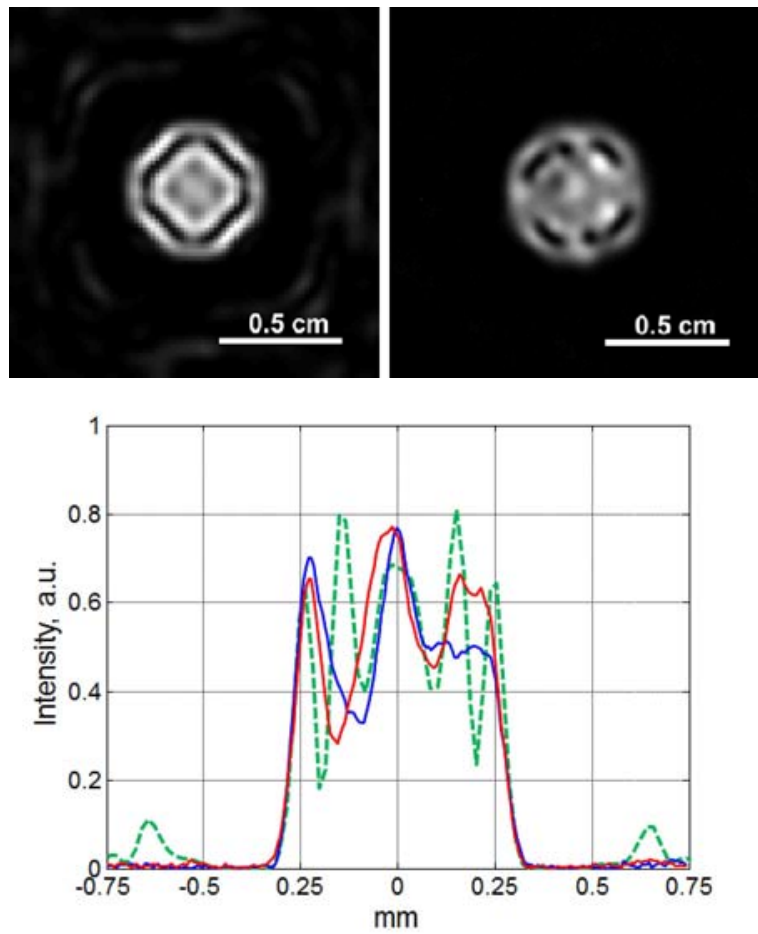

Fig. 4 (a) Simulated (left) and measured (right) laser beam profiles in the focal plane of a $400 \mathrm{~mm}$ lens shaped with a binary phase only hologram. (b) Intensity outlines for the simulated (dashed green) and measured (blue line - horizontal, red line - vertical) beam profiles.

The matching size and shape of the ablated $(14.5 \mu \mathrm{m})$ and design $(14 \mu \mathrm{m})$ pixels, high diffraction efficiency (combined power in the shaped beam constitutes $77 \%$ of the total vs expected $81 \%$ ), and the close resemblance of 
the simulated and the measured beam profiles confirm that the hologram was produced with a very high degree of exactness.

\section{Beam shaping with multi-level phase only holo- grams}

In general, the closeness of the simulated flat top beam profile to the desired shape strongly depends on the ratio $\beta$ of the targeted beam radius to the radius of the focal spot of the corresponding Gaussian beam (focused without the beam shaping element) [15] and the number of the phase shift levels $n$ that can be introduced by a DOE. In the considered example $\beta \sim 6$, which is sufficiently large and should not lead to a substantial deviation from the targeted uniform focal intensity distribution. In the presented case, the apparent deviation from the flat-top profile seen in both the simulated and the measured beams should be attributed to the fundamental limitations of binary holograms. As an intermediate conclusion, further development of ITO nanoparticulate based phase only hologram should be directed towards development of multi-level diffractive optical elements.

Multi-level DOEs are capable of introducing phase shifts with an increment of $2 \pi / n$, where $n$ is the number of phase levels. For binary holograms $n=2$ and only two levels $(0, \pi)$ are feasible. The number of levels strongly affects performance of phase only holograms in terms of diffraction efficiency and complexity of possible patterns. In this case determination of a proper number of phase levels is an important task in order to achieve an acceptable compromise between the hologram performance and the fabrication complexity. According to [14] a binary diffraction grating will have the efficiency of $40.5 \%$ for either order, with 4 levels the efficiency rises to $81 \%$, with 8 levels to $95 \%$, with 16 levels to $99 \%$, and $100 \%$ efficiency can be achieved with continuous phase variation.
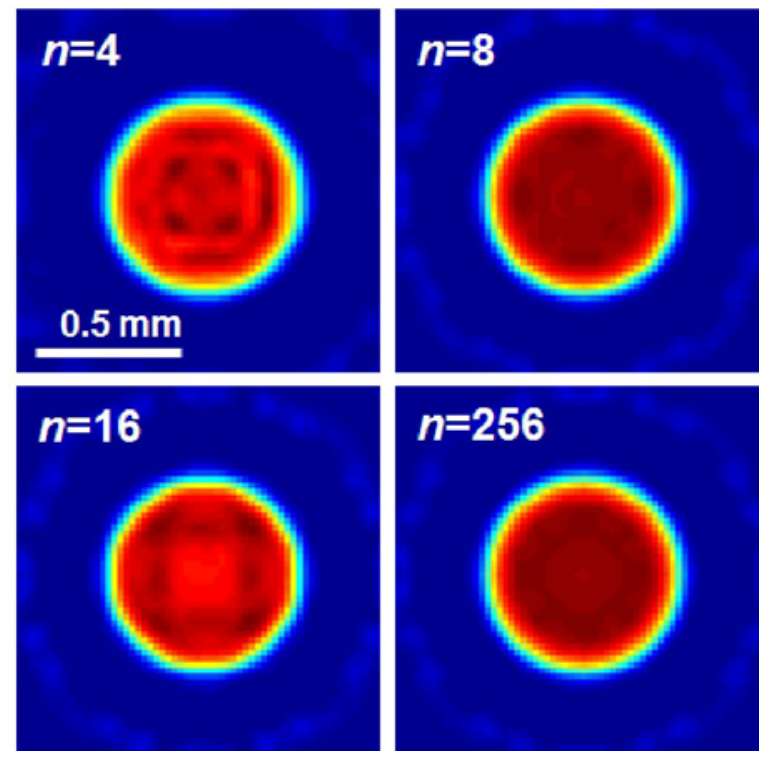

Fig. 5 Simulated beam intensity distribution for $n=4,8$, 16, 256 phase levels. The targeted shape is a super Gaussian beam with $m=10$ and $1 / e^{2}$ radius of $300 \mu \mathrm{m}$. For $n=2$ the intensity distribution is shown in Fig. 4a.
Fig. 5 shows how the beam intensity distribution is expected to change for $4,8,16,256$ phase levels. The targeted intensity distribution is identical to the one described earlier in the paper and the case of $n=2$ is shown in Fig. 4a. Based on the simulation results as well the stated diffraction efficiencies $n=8$ appears to be an optimal compromise between the hologram and fabrication complexity. Although increasing the number of levels from only 2 to 4 will provide the largest gain in terms of the absolute diffraction efficiency and should be considered first.

Fabrication of multi-level holograms based on the selective ultrafast laser ablation of ITO nanoparticulate layers appears to be feasible due to the fact that annealing of such layers not only widens the transmission window but also increases their laser ablation threshold approximately by a factor of 2 [16]. If the laser fluence is adjusted to be above the ablation threshold of non-annealed but below the ablation threshold of annealed layers, then a pixel can be created not only on top of the glass substrate but also above an annealed surface. The concept of fabrication of multi-level holograms is shown in Fig. 6 and it includes multiple repetitions of the following cycle: coating to form a new layer, selective ablation of non-annealed areas based on the hologram design, and subsequent annealing to increase the resistivity of the fabricated part to the ablation.

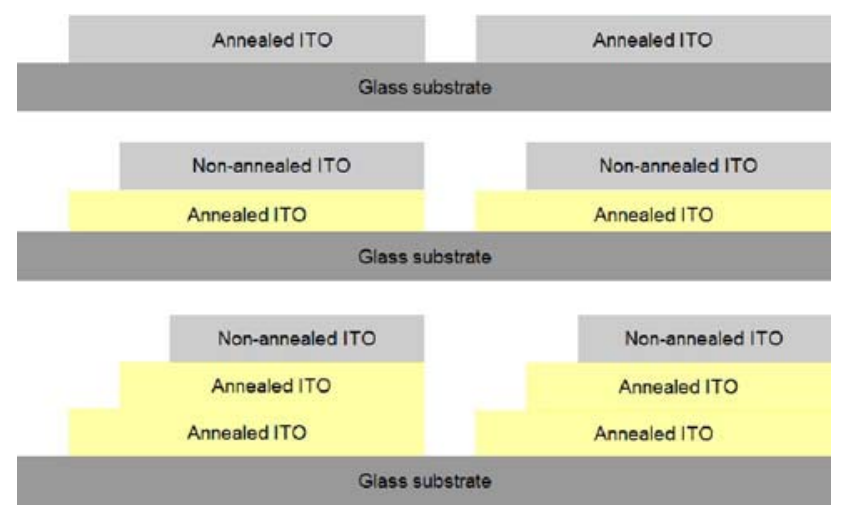

Fig. 6 Fabrication steps for multi-level ITO nanoparticle based phase only holograms .

At the first cycle a bare glass substrate is coated with an ITO nanoparticulate layer of thickness $T=\lambda / n /\left(n_{\lambda}-1\right)$, where $n$ is the number of phase levels and $n_{\lambda}$ is the refractive index at the design wavelength $\lambda$. Then, the pixels with a phase shift of 0 are ablated. At the next step the hologram is annealed and after that recoated with the same layer thickness. The non-annealed material is partly ablated to restore 0 phase shift pixels and to form level 2. Effectively, the laser ablation is used not only to form new pixels but also to restore the ones that have been already produced and subsequently refilled during the spin-coating. In this concept the number of coating-ablation-annealing steps that are required to fabricate a hologram becomes $n-1$. Fig. 7 shows a multistep profile successfully produced using the above described methodology. The shown profile demonstrates that multi-level holograms can be fabricated using sequential ablation of non-annealed ITO nanoparticles. On the other hand the same profile also underlines several challenges that have to be overcome. The ablated surfaces 
of the $1^{\text {st }}$ and the $2^{\text {nd }}$ steps are rougher than the glass substrate or the last coating layer presumably due to the residual ITO material not completely removed during the ablation. This issue is likely can be addressed via more accurate fine-tuning of the laser parameters such as pulse energy, spot size, and the number of pulses per unit area. Additionally, since the hologram fabrication includes repeating steps the issue of layer to layer registration becomes present. Although this problem has been solved for a number of applications (e.g., photolithography) it still remains technically challenging and has to be addressed by the authors.

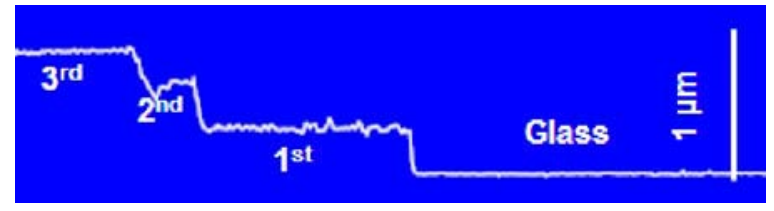

Fig. 7 Profile of multi-step structure fabricated by subsequent ablation of non-annealed ITO nanoparticulate layers.

\section{Conclusion}

It has been demonstrated that high quality binary phase only holograms can be fabricated by spatially selective ablation of ITO nanoparticulate coatings of proper thicknesses. The performance of such holograms has been shown to be very close to the theoretically predicted. It has been verified that ITO coatings can withstand high cw laser power $\left(3.9 \mathrm{~kW}\right.$ total with the peak intensity of $\left.4 \mathrm{~kW} / \mathrm{cm}^{2}\right)$ at a wavelength of $1.030 \mu \mathrm{m}$ making such holograms of interest for beam shaping of high power solid state or fiber lasers. Further development of the holograms should include fabrication of multi-level structures with the number of levels at least doubled compared to the binary ones. Such multi-level holograms can be produced via multiple cycles of coating, ultrafast laser ablation, and annealing of the deposited ITO layers. However the surface roughness of the intermediate levels has to be reduced as well as the issue of registering sequentially ablated layers has to be resolved too.

\section{Acknowledgements}

The authors gratefully acknowledge funding of the project within the priority program 1327 "Optisch erzeugte Sub-100-nm-Strukturen für biomedizinische und technische Applikationen" and funding of the Erlangen Graduate School in Advanced Optical Technologies (SAOT) both by the German National Science Foundation (DFG).

\section{References}

[1] E. Buckley: J. Display Technol. 7, (2011) 135.

[2] L. Sacconi, E. Froner, R. Antolini, M.R. Taghizadeh, A. Choudhury, F.S. Pavone: Opt. Lett. 28, (2003) 1918.

[3] J.A. Neff, R.A. Athale, S.H. Lee: Proc. IEEE 78, (1990) 826

[4] S.J. Walker, J. Jahns: J. Opt. Soc. Am. A 7, (1990) 1509.

[5] C.G. Blough, M. Rossi, S.K. Mack, R.L. Michaels: Appl. Opt. 36, (1997) 4648.

[6] C. Falldorf, C. Dankwart, R. Gläbe, B. Lünemann, C. V. Kopylow, R.B. Bergmann: Applied optics 48, (2009)
5782.

[7] V. Kalima, J. Pietarinen, S. Siitonen, J. Immonen, M. Suvanto, M. Kuittinen, K. Mönkkönen, T.T. Pakkanen: Optical Materials 30, (2007) 285.

[8] M.T. Gale, C. Gimkiewicz, S. Obi, M. Schnieper, J. Söchtig, H. Thiele, S. Westenhöfer: Optics in Switzerland 43, (2005)373.

[9] M.T. Flores-Arias, A. Castelo, C. Gomez-Reino, G.F. de la Fuente: Optics Communications 282, (2009) 1175.

[10]M. Baum, J. Strauß, F. Grüßel, I. Alexeev, M. Schmidt: J. Opt. 16, (2014) 125706.

[11]M. Baum, I. Alexeev, M. Latzel, S.H. Christiansen, M. Schmidt: Opt. Express 21, (2013) 22754.

[12]P. Amend, O. Hentschel, C. Scheitler, M. Baum, J. Heberle, S. Roth, M. Schmidt: Laser Micro/Nanoengin. 8 (2013) 276.

[13]M. Gross, A. Winnacker, P.J. Wellmann: First International Symposium on Transparent Conducting Oxides 515, (2007) 8567

[14]M.W. Farn, W.B. Veldkamp: "Handbook of Optics" 2, (1995) 8.1

[15]L.A. Romero, F.M. Dicke: J. Opt. Soc. Am. A 13, (1996) 751.

[16]F. Mikschl, M. Baum, J. Heberle, I. Alexeev, M. Schmidt: Physics Procedia 56, (2014) 991.

(Received: May 22, 2015, Accepted: July 7, 2016) 